\title{
ON THE DYNAMICS OF A FAMILY OF THIRD-ORDER ITERATIVE FUNCTIONS
}

\author{
SERGIO AMAT ${ }^{1}$, SONIA BUSQUIER ${ }^{1}$ and SERGIO PLAZA $\cong 2$
}

(Received 4 December, 2003; revised 5 May, 2006)

\begin{abstract}
We study the dynamics of a family of third-order iterative methods that are used to find roots of nonlinear equations applied to complex polynomials of degrees three and four. This family includes, as particular cases, the Chebyshev, the Halley and the super-Halley root-finding algorithms, as well as the so-called c-methods. The conjugacy classes of these iterative methods are found explicitly.
\end{abstract}

2000 Mathematics subject classification: primary 37N30; secondary $65 \mathrm{~F} 10,30 \mathrm{C} 15$.

Keywords and phrases: iteration functions, dynamics, rational maps, conjugacy classes.

\section{Introduction}

One of the most important applications of an iterative method is the search for roots of a nonlinear equation $f(z)=0$. We begin with an initial guess $z_{0}$, which is then improved by means of an iterative function $\Phi$, that is, $z_{n+1}=\Phi\left(z_{n}\right)$. Conditions are imposed on $z_{0}$ (and, eventually, on $f$ or $\Phi$ ) to ensure the convergence of the sequence of iterates $\left(z_{n}\right)_{n \geq 0}$ to a solution $z^{*}$ of the equation $f(z)=0$, as well as to establish the order of convergence of the iterative method defined by $\Phi$.

For example, Newton's root-finding algorithm

$$
z_{n+1}=z_{n}-\frac{f\left(z_{n}\right)}{f^{\prime}\left(z_{n}\right)}=N_{f}\left(z_{n}\right)
$$

and other second-order root-finding algorithms have been extensively used and studied, see [30] and the references therein. For the polynomial equations $p(z)=0$, the

\footnotetext{
'Departamento de Matemática Aplicada y Estadística. Universidad Politécnica de Cartagena, Spain; e-mail: sergio.amat@upct.es and sonia.busquier@upct.es.

${ }^{2}$ Depto. de Matemáticas, Facultad de Ciencias, Universidad de Santiago de Chile, Casilla 307,

Correo 2, Santiago, Chile; e-mail: splaza@lauca.usach.cl.

(C) Australian Mathematical Society 2007, Serial-fee code 1446-1811/07
} 
iterative function $N_{p}$ defines a rational mapping on the Riemann sphere $\overline{\mathbb{C}}=\mathbb{C} \cup\{\infty\}$. The simple roots of $p$, that is, the roots that are not critical points are superattracting fixed points of $N_{p}$, which means that, if $z^{*}$ is a simple root of $p$, then $N_{p}\left(z^{*}\right)=z^{*}$ and $N_{p}^{\prime}\left(z^{*}\right)=0$. This implies that if $z_{0}$ is chosen conveniently near $z^{*}$, then the sequence $z_{n+1}=N_{p}\left(z_{n}\right)$ converges quadratically to $z^{*}$. For the dynamics of Newton's method, see [30].

Although third-order root-finding algorithms require more computational cost than simpler algorithms, in some cases they are preferred on account of greater speed of convergence of the sequence iterates when the initial guess is chosen conveniently. For instance, these algorithms have been successfully used in the search for solutions of nonlinear integral equations $[11,12]$. They have also been used to solve quadratic equations in a very fast way [3]. On the other hand, third-order root-finding algorithms are also interesting from a theoretical point of view because they provide a variety of results on existence and uniqueness of solutions that improve, by and large, the results obtained by using Newton-type algorithms, see [25] or [6].

In this paper, we are interested in understanding a family of classical third-order root-finding algorithms from the point of view of their dynamics.

The paper is organized as follows. In Section 2, we review the basic notions on complex dynamics to be used. For a comprehensive study of the theory of iteration of rational maps, see [9], [28] and [29]. In Section 3, we introduce a general family of third-order root-finding algorithms whose dynamics we are interested in understanding. These root-finding algorithms are induced by iterative methods. The conjugacy classes of the family of iterative methods presented in Section 3 are constructed in Section 4. Finally, in Section 5, the equations of the critical points and additional fixed points of the iterative methods under study are obtained.

\section{Basic notions}

Before presenting the iterative methods we are interested in, we shall recall some basic notions of complex dynamics. Let

$$
R(z)=\frac{P(z)}{Q(z)}
$$

be a rational map on the Riemann sphere, where $P$ and $Q$ are complex polynomials with no common factors. We say that $z_{0}$ is a fixed point of $R$ if $R\left(z_{0}\right)=z_{0}$. For $z \in \overline{\mathbb{C}}$, we define its forward orbit as the set $\operatorname{orb}(z)=\left\{z, R(z), R^{2}(z), \ldots, R^{k}(z), \ldots\right\}$ where $R^{k}$, for $k \in \mathbb{N}$, means the $k$-fold iterate of $R$. A periodic point of period $n$ is a point $z_{0}$ such that $R^{n}\left(z_{0}\right)=z_{0}$ and $R^{j}\left(z_{0}\right) \neq z_{0}$ for $0<j<n$. Observe that if $z_{0} \in \overline{\mathbb{C}}$ is a periodic point of period $n \geq 1$, then $z_{0}$ is a fixed point of $R^{n}$. Also, recall that 
a fixed point $z_{0}$ is, respectively, attracting, repelling or indifferent in the case where $\left|R^{\prime}\left(z_{0}\right)\right|$ is less than, greater than, or equal to 1 . A periodic point of period $n$ is said to be attracting, repelling or indifferent if, as a fixed point of $R^{n}$, it is respectively attracting, repelling or indifferent. A superattracting fixed point of $R$ is a fixed point which is also a zero of the derivative $R^{\prime}$. A periodic point of period $n$ is said to be a superattracting periodic point of $R$ if, as a fixed point of $R^{n}$, it is superattracting.

Let $\zeta$ be an attracting fixed point of $R$. The basin of attraction of $\zeta$ is the set $B(\zeta)=\left\{z \in \overline{\mathbb{C}}: R^{n}(z) \rightarrow \zeta\right.$ as $\left.n \rightarrow \infty\right\}$. The immediate basin of attraction of an attracting fixed point $\zeta$ of $R$, denoted $B^{*}(\zeta)$, is the connected component of $B(\zeta)$ containing $\zeta$. Finally, if $z_{0}$ is an attracting periodic point of period $n$ of $R$, the basin of attraction of the orbit orb $\left(z_{0}\right)$ is the set $B\left(\operatorname{orb}\left(z_{0}\right)\right)=\bigcup_{j=0}^{n-1} R^{j}\left(B\left(z_{0}\right)\right)$, where $B\left(z_{0}\right)$ is the attraction basin of $z_{0}$ as a fixed point of $R^{n}$. The Julia set of a rational map $R$, denoted $\mathscr{J}(R)$, is the closure of the set of repelling periodic points. Its complement is the Fatou set $\mathscr{F}(R)$. If $R$ has an attracting. fixed point $z_{0}$, then the basin of attraction $B\left(z_{0}\right)$ is contained in the Fatou set and $\mathscr{J}(R)=\partial B\left(z_{0}\right)$. Therefore, the chaotic dynamics of $R$ are contained in its Julia set (see [9]).

In what follows, we will assume that $f: U \rightarrow \mathbb{C}$ is an analytic function where $U \subset \mathbb{C}$ is an open set. Our main interest is the case where $U=\mathbb{C}$ and $f$ is a polynomial function.

DEFINITION 1 . We say that a map $f \rightarrow T_{f}$ carrying a complex-valued function $f$ to a function $T_{f}: \overline{\mathbb{C}} \rightarrow \overline{\mathbb{C}}$ is a one-point iterative root-finding algorithm if every root of $f$ is an attracting fixed point of $T_{f}$. It is convergent if, given an initial guess $z_{0}$, the sequence of iterates $\left(z_{k}\right)_{k \geq 0}$, where $z_{k+1}=T_{f}\left(z_{k}\right)$, converges to a root $z^{*} \in \overline{\mathbb{C}}$ of $f$ whenever $z_{0}$ is sufficiently close to $z^{*}$.

\section{The third-order family}

We consider the family of iterative root-finding algorithms given for $n \geq 0$ by

$$
z_{n+1}=M_{f, \theta, c}\left(z_{n}\right)=z_{n}-\left(1+\frac{L_{f}\left(z_{n}\right)}{2\left(1-\theta L_{f}\left(z_{n}\right)\right)}+c\left[L_{f}\left(z_{n}\right)\right]^{2}\right) u_{f}\left(z_{n}\right)
$$

where $z_{0}$ is an initial guess, $L_{f}(z)=f(z) f^{\prime \prime}(z) / f^{\prime}(z)^{2}, u_{f}(z)=f(z) / f^{\prime}(z)$, if $f^{\prime}(z) \neq 0$, and $\theta, c$ are complex parameters, both to be chosen conveniently in each case. This iterative root-finding algorithm is induced by the family of iterative functions

$$
M_{f, \theta, c}(z)=z-\left(1+\frac{L_{f}(z)}{2\left(1-\theta L_{f}(z)\right)}+c\left[L_{f}(z)\right]^{2}\right) u_{f}(z)
$$


Observe that when we apply any of these iterative functions (3.1) to a polynomial, we obtain a rational map on the Riemann sphere.

This family of third-order iterative algorithms includes, as particular cases, the following (see [2]).

(1) When $c=0$ and the parameter $\theta$ is real and nonnegative, we obtain the family of third-order iterative functions $M_{f, \theta}(z)=M_{f, \theta, 0}(z)$ studied in [23]

$$
M_{f, \theta}(z)=z-\left(1+\frac{L_{f}(z)}{2\left(1-\theta L_{f}(z)\right)}\right) u_{f}(z)
$$

In particular, we have that

(a) The well-known Chebyshev iteration function

$$
C_{f}(z)=z-\left(1+\frac{1}{2} L_{f}(z)\right) u_{f}(z)
$$

is obtained from family (3.2) when $\theta=0$.

(b) The well-known Halley iterative method

$$
H_{f}(z)=z-\left(\frac{2}{2-L_{f}(z)}\right) u_{f}(z)=z-\frac{2 f(z) f^{\prime}(z)}{2 f^{\prime}(z)^{2}-f(z) f^{\prime \prime}(z)}
$$

is obtained from family (3.2) when $\theta=1 / 2$. The dynamics of Halley's iterative method have been studied in [10] and [31].

(c) Another third-order iterative method perhaps not as well known as the previous two is the so-called super-Halley iterative method (see [24]), which is given by

$$
S H_{f}(z)=z-\left(1+\frac{L_{f}(z)}{2\left(1-L_{f}(z)\right)}\right) u_{f}(z)
$$

and which is obtained from family (3.2) when $\theta=1$.

(2) When $c$ is a nonzero parameter and $\theta=0$, we obtain the so-called $c$-iterative methods

$$
M_{f, c}(z)=z-\left(1+\frac{1}{2} L_{f}(z)+c L_{f}(z)^{2}\right) u_{f}(z) .
$$

This class of iterative methods has been introduced in [1] and [22], where we can find a study of their convergence for $0 \leq c \leq 2$.

Many papers have been published on the convergence of third-order iterative rootfinding algorithms. Thus, for Chebyshev's method we have [7, 12, 26]; for Halley's method [11, 15]; for the super-Halley method [16, 24], for the $c$-methods [1]; as well as for Laguerre's iterative root-finding algorithm, [20] and the references therein. 


\section{Conjugacy classes}

We recall the definition of conjugacy.

DEFINITION 2. Let $R_{1}, R_{2}: \overline{\mathbb{C}} \rightarrow \overline{\mathbb{C}}$ be two rational maps. We say that $R_{1}$ and $R_{2}$ are conjugated if there exists a Möbius transformation (an analytic homeomorphism of the Riemann sphere) $\phi: \overline{\mathbb{C}} \rightarrow \overline{\mathbb{C}}$ such that $R_{2} \circ \phi(z)=\phi \circ R_{1}(z)$, for all $z \in \overline{\mathbb{C}}$.

The maps $R_{1}$ and $R_{2}$ can be regarded as the same map viewed in different coordinate systems.

An important feature of conjugation of rational maps is given by the following classical result.

THEOREM 4.1. Let $R_{1}$ and $R_{2}$ be two rational maps, and let $\phi$ be a Möbius transformation conjugating $R_{1}$ and $R_{2}$, that is, $R_{2}=\phi \circ R_{1} \circ \phi^{-1}$. Then $\mathscr{F}\left(R_{2}\right)=\phi\left(\mathscr{F}\left(R_{1}\right)\right)$ and $\mathscr{J}\left(R_{2}\right)=\phi\left(\mathscr{J}\left(R_{1}\right)\right)$.

Conjugacy plays a central role in understanding the behaviour of classes of maps from the point of view of dynamical systems in the following sense. Suppose that one wishes to describe both the quantitative and the qualitative behaviour of a map $z \mapsto \Phi_{f}(z)$, where $\Phi_{f}$ is some iteration function as in Section 3. Since conjugacy preserves fixed and periodic points and their types as well as basins of attraction, the dynamical features concerning $f$ are carried by the fixed points of $\Phi_{f}$ as well as by the nature of such fixed points which may be (super)attracting, repelling or indifferent. Therefore, it is worthwhile to build up, for polynomials of degree two and three, a parameterized family consisting of polynomials which are as simple as possible such that a conjugacy exists between the corresponding iterative methods.

For the family of iterative methods (3.1), we begin with a useful result.

THEOREM 4.2 (Scaling). Let $f(z)$ be an analytic map on the Riemann sphere, and let $T(z)=\alpha z+\beta$, with $\alpha \neq 0$, be an affine map. If $g(z)=\lambda(f \circ T(z))$, where $\lambda \in \mathbb{C}$ is a nonzero constant, then $T \circ M_{g, \theta, c} \circ T^{-1}(z)=M_{f, \theta, c}(z)$. That is, $M_{f, \theta, c}$ and $M_{g, \theta, c}$ are conjugated by $T$.

\section{Proof. See [4].}

The scaling theorem allows us, via suitable changes of coordinates, to reduce the study of the dynamics of iterative methods $M_{f, \theta, c}$ to the study of specific families of iteration functions of simpler maps.

We recall that a polynomial is said to be generic when all of its roots are simple. 
4.1. Quadratic polynomials It is well known that every quadratic polynomial $p(z)=\alpha z^{2}+2 \beta z+\gamma(\alpha \neq 0)$, reduces, via the affine change of coordinates $L(z)=(z-\beta) / \alpha$, to the polynomial $p_{\mu}(z)=z^{2}-\mu$, where $\mu=\beta^{2}-\alpha \gamma-\beta$, that is, any quadratic polynomial, via an affine change of coordinates, reduces to a polynomial belonging to the one-parameter family of quadratic polynomials $\left\{p_{\mu}\right\}_{\mu}$. Note that $p_{\mu}$ is generic if $\mu \neq 0$. Now we have the following result.

THEOREM 4.3. Let $p(z)=\alpha z^{2}+2 \beta z+\gamma$, with $\alpha \neq 0$, be a generic quadratic polynomial, and let $p_{\mu}(z)=z^{2}-\mu$, where $\mu=\beta^{2}-\alpha \gamma-\beta$. Then the affine scaling of coordinates $L(z)=(z-\beta) \alpha$ provides a conjugacy between $M_{p, \theta, c}$ and $M_{p_{\mu}, \theta, c}$.

PROOF. It is straightforward.

REMARK. For the non-generic polynomial $p(z)=z^{2}$, the iterative method $M_{p, \theta, c}(z)$ reduces to the simpler linear iterative method

$$
G(z)=\frac{z(6-4 \theta-2 c+c \theta)}{8(\theta-2)}
$$

which has $z_{0}=0$ as its unique attracting fixed point.

DEFINITION 3 (Universal Julia set). We say that an iterative root-finding algorithm $f \rightarrow T_{f}$ has a universal Julia set for polynomials of degree $d$ if there exists a rational map $R$ such that for every polynomial $f$ of degree $d, \mathscr{J}\left(T_{f}\right)$ is conjugated by a Möbius transformation $\phi$ to $\mathscr{J}(R)$, that is, $\mathscr{J}\left(T_{f}\right)=\phi(\mathscr{J}(R))$.

The result that follows, due to Cayley [13, 14] and to Schröder [32], has great historical importance. In an attempt to understand the dynamics of Newton's method in the complex plane, Cayley investigated the dynamics of Newton's method applied to polynomials of a particularly simple form. He realized that major difficulties would arise when attempting to extend the following result for quadratics to cubics and beyond. It is believed that this circumstance motivated further work of P. Fatou and G. Julia along these lines.

THEOREM 4.4. Let

$$
N_{p}(z)=\frac{z^{2}-a b}{2 z-(b+a)}
$$

be the rational map obtained from Newton's method applied to a generic quadratic polynomial $p(z)=(z-a)(z-b)$. Then $N_{p}$ is conjugated to the map $R(z)=z^{2}$ by the Möbius transformation $M(z)=(z-a) /(z-b)$, that is, $R(z)=M \circ N_{p} \circ M^{-1}(z)$. Moreover, $\mathscr{J}\left(N_{p}\right)$ is the straight line in the complex plane corresponding to the locus of points equidistant from $a$ and $b$. 
It follows from Theorem 4.4 that the Julia set of Newton's algorithm applied to a quadratic polynomial, up to a change of coordinates, is the unit circle in the complex plane. That is, Newton's iteration function has a universal Julia set when it is applied to quadratic polynomials.

We also have extensions of this theorem due to K. Kneisl.

THEOREM 4.5 (See [27]). Let

$$
H_{p}(z)=\frac{z^{3}-3 a b z+a b(a+b)}{3 z^{2}-3(a+b) z+a^{2}+a b+b^{2}}
$$

be the rational map obtained from Halley's iterative root-finding algorithm applied to a generic quadratic polynomial $p(z)=(z-a)(z-b)$. Then $H_{p}$ is conjugated to the map $Q(z)=z^{3}$ by the Möbius transformation $M(z)=(z-a) /(z-b)$, that is, $Q(z)=M \circ H_{p} \circ M^{-1}(z)$. Moreover, $\mathscr{J}\left(H_{p}\right)$ is the straight line in the complex plane corresponding to the locus of points equidistant from $a$ and $b$.

Theorem 4.5 shows that the Julia set of Halley's iterative root-finding algorithm applied to a quadratic polynomial, up to a change of coordinates, is the unit circle in the complex plane. That is, Halley's iterative method has a universal Julia set when it is applied to quadratic polynomials.

For Chebyshev's iterative root-finding algorithm, also known as the super-Newton algorithm, the following result holds.

THEOREM 4.6 (See [27]). Let

$$
C_{p}(z)=\frac{3 z^{4}-2(a+b) z^{3}-6 a b z^{2}+6 a b(a+b) z-a b\left(a^{2}+3 a b+b^{2}\right)}{(2 z-a-b)^{3}}
$$

be the rational map obtained from the super-Newton algorithm applied to a generic quadratic polynomial $p(z)=(z-a)(z-b)$. Then $C_{p}$ is conjugated to the map $S_{3}(z)=\left(z^{4}+2 z^{3}\right) /(2 z+1)$ via the Möbius transformation $M(z)=(z-a) /(z-b)$. That is, $S_{3}(z)=M \circ C_{p} \circ M^{-1}(z)$.

Theorem 4.6 shows that the Julia set of Chebyshev's iterative root-finding algorithm applied to a quadratic polynomial, up to a change of coordinates, is the same as that of the rational map $S_{3}(z)=\left(z^{4}+2 z^{3}\right) /(2 z+1)$. This means that Chebyshev's iterative method has a universal Julia set when it is applied to quadratic polynomials.

Now we have the following extension of the above theorems.

THEOREM 4.7. The rational map obtained from the $M_{f, \theta, c}(z)$ algorithm applied to a generic quadratic polynomial $p(z)=(z-a)(z-b)$ is conjugated to the rational 
map

$$
\begin{aligned}
R(z)= & z^{3}\left(z^{5}+(6-2 \theta) z^{4}+(14-8 \theta) z^{3}+(16-4 c-12 \theta) z^{2}\right. \\
& +(9-8 c-8 \theta+8 c \theta) z+2-4 c-2 \theta) /\left((2-4 c-2 \theta) z^{5}\right. \\
& +(9-8 c-8 \theta+8 c \theta) z^{4}+(16-4 c-12 \theta) z^{3} \\
& \left.+(14-8 \theta) z^{2}+(6-2 \theta) z+1\right)
\end{aligned}
$$

via the Möbius transformation $M(z)=(z-a) /(z-b)$. In particular, for the superHalley algorithm we obtain the simplest rational map $T(z)=z^{4}$, and for the $c$ algorithms we obtain the following rational map:

$$
R(z)=\frac{z^{3}\left(z^{3}+4 z^{2}+5 z+2-4 c\right)}{(2-4 c) z^{3}+5 z^{2}+4 z+1} .
$$

The proof of Theorem 4.7 is straightforward.

Theorem 4.7 shows that the Julia set of the super-Halley iterative root-finding algorithm applied to a quadratic polynomial, up to a change of coordinates, is the unit circle in the complex plane. This means that the super-Halley iteration function has a universal Julia set when it is applied to quadratic polynomials. The $c$-algorithms also have the properties of a universal Julia set when they are applied to quadratic polynomials.

\subsection{Cubic polynomials For cubic polynomials we have the following result.}

THEOREM 4.8. Let $p(z)=\left(z-z_{0}\right)\left(z-z_{1}\right)\left(z-z_{2}\right)$ be a generic cubic polynomial with roots ordered as follows: $0 \leq\left|z_{0}\right| \leq\left|z_{1}\right| \leq\left|z_{2}\right|$. Let $T(z)=\left(z_{2}-z_{0}\right) z+z_{0}$ and $\lambda=\left(z_{1}-z_{0}\right) /\left(z_{2}-z_{0}\right)$. Using the affine change of coordinates $T$, the polynomial $p$ reduces to an element belonging to the parameterized family $\left\{q_{\rho}\right\}_{\rho \in \mathbb{C}}$, where $q_{\rho}(z)=$ $z(z-1)(z-\rho)$. Moreover, $T$ is a conjugacy between $M_{p . \theta, c}$ and $M_{q_{\lambda}, \theta, c}$, or in other words, $T^{-1} \circ M_{q_{\lambda}, \theta, c} \circ T=M_{p, \theta, c}$.

The proof of Theorem 4.8 is straightforward.

It is easy to show that the one-parameter family $q_{\rho}(z)=z(z-1)(z-\rho)$ reduces to the well-known one-parameter family $p_{A}(z)=z^{3}+(A-1) z-A$ (see [17]) by an affine change of coordinates. Therefore, we have reduced the study of the dynamics of $M_{q_{\rho}, \theta, c}$ to the study of the dynamics of $M_{p_{A}, \theta, c}$, and since every cubic polynomial $p(z)=a z^{3}+b z^{2}+c z+d$ reduces, via an affine change of coordinates, to a polynomial belonging to either the one-parameter family $p_{A}$ for a suitable choice of the parameter $A$ or to $z^{3}$, it follows that an appropriate scaling puts $M_{p, \theta, c}$ inside the conjugacy class of $M_{Q, \theta, c}$, where $Q$ stands for either $p_{A}$ or $z^{3}$ in each case. Now for the polynomial $p(z)=z^{3}$, we have

$$
M_{p . \theta . c}(z)=\frac{z(45-12 c-36 \theta+8 c \theta)}{27(3-2 \theta)} .
$$


The relationship between the parameter $A$ and the parameter $\rho$ is given above and the relationship between the parameter $\rho$ and the roots of the original polynomial is given in the statement of the theorem. A study of the $A$-parameter space for Newton's iterative method associated to the one-parameter family $p_{A}$ can be found in [17]. Recall that König's iterative method applied to a complex function $f$ is given by

$$
K_{f, \sigma}(z)=z+(\sigma-1) \frac{(1 / f(z))^{(\sigma-2)}}{(1 / f(z))^{(\sigma-1)}}, \quad(\sigma=2,3, \ldots)
$$

This is a family of iterative methods depending on the parameter $\sigma$. It includes Halley's iterative method as a member. A fair amount of progress in the study of the $A$-parameter space when this family of root-finding algorithms is applied to the cubic polynomials $p_{A}$ has been achieved in $[5,33,34]$.

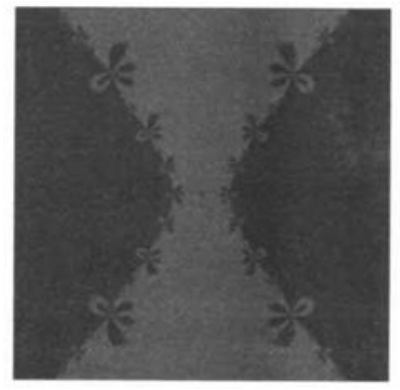

$A=0$

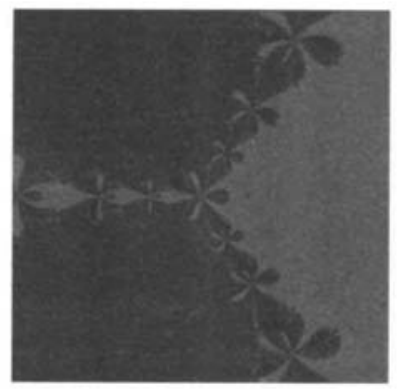

$A=1$

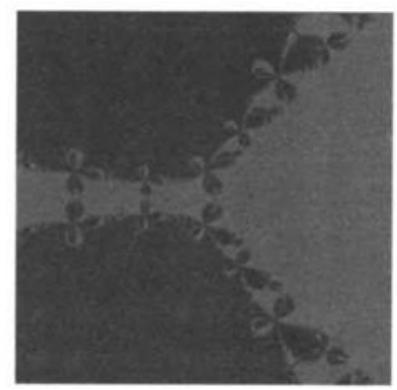

$A=2$

FIGURE 1. Attraction basins of the roots of $p_{A}$ and the Julia set of the rational obtained when Chebyshev's iterative method is applied to $p_{A}$.

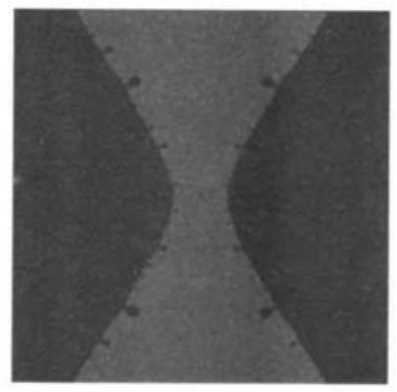

$A=0$

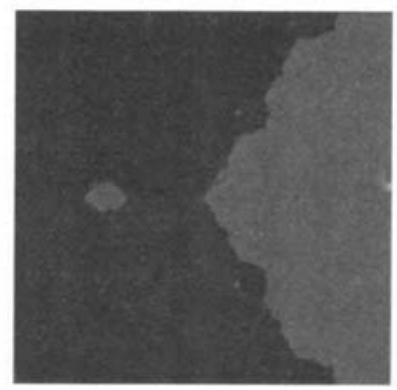

$A=1$

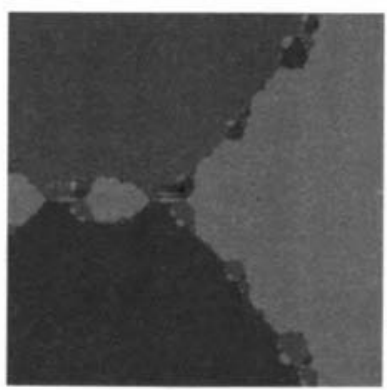

$A=2$

FIGURE 2. Attraction basins of the roots of $p_{A}$ and the Julia set of the rational obtained when Halley's iterative method is applied to $p_{A}$. 


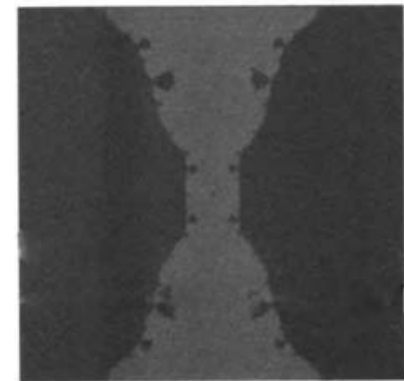

$A=0$

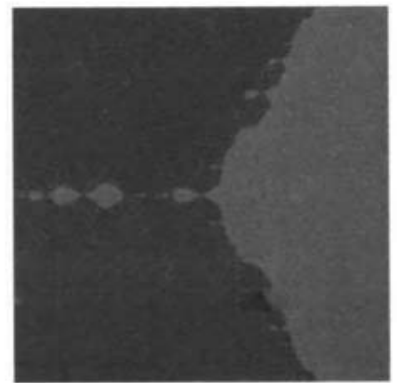

$A=1$

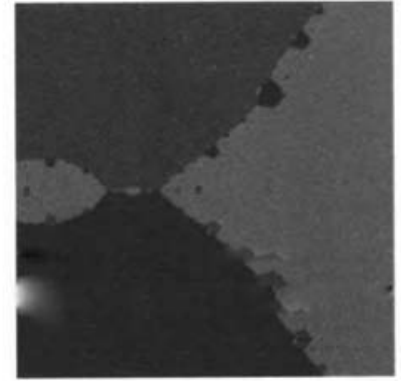

$A=2$

FIGURE 3. Attraction basins of the roots of $p_{A}$ and the Julia set of the rational obtained when the super-Halley iterative method is applied to $p_{A}$.
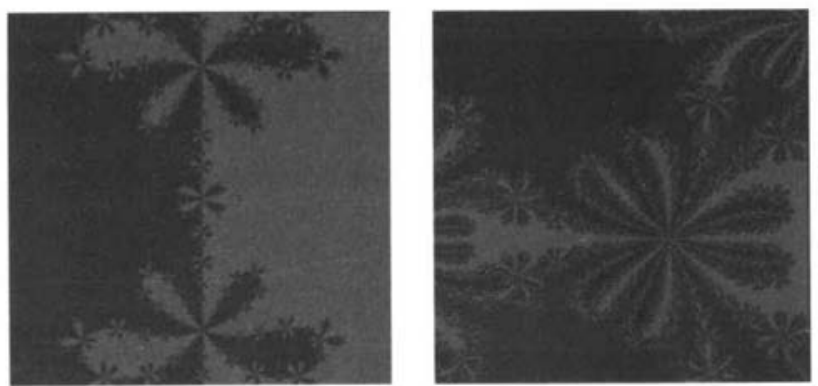

FIGURE 4. Attraction basins of the roots of $p_{\mu}$ and of $p_{A}$, and the corresponding Julia set when the $c$-method, for $c=2$, is applied to $p_{\mu}$, with $\mu=2$; and to $p_{A}$, with $A=1$, respectively.

4.3. Quartic polynomials In the case of quartic polynomials, the following result holds.

THEOREM 4.9. Let $p(z)=\left(z-z_{0}\right)\left(z-z_{1}\right)\left(z-z_{2}\right)\left(z-z_{4}\right)$ be a degree four generic polynomial having its roots ordered as follows: $0 \leq\left|z_{0}\right| \leq\left|z_{1}\right| \leq\left|z_{2}\right| \leq\left|z_{3}\right|$. Let $\eta=\left(z_{1}-z_{0}\right) /\left(z_{3}-z_{0}\right)$ and $\zeta=\left(z_{2}-z_{0}\right) /\left(z_{3}-z_{0}\right)$. Using the affine change of coordinates $T$, the polynomial $p$ reduces to an element belonging to the parameterized family of quartic polynomials $\left\{q_{\rho, \mu}\right\}_{\rho, \mu \in \mathrm{C}}$ where $q_{\rho, \mu}(z)=z(z-1)(z-\rho)(z-\mu)$. Moreover, $T$ is a conjugacy between $M_{p, \theta, c}$ and $M_{q_{n, s}, \theta, c}$.

The proof of Theorem 4.9 is straightforward.

The degree four case is somewhat less well understood. However, some computational progress has been made concerning the description of the parameter space for the family of polynomials $p_{\lambda}(z)=z^{4}+(\lambda-1) z^{2}-\lambda=(z-1)(z+1)\left(z^{2}+\lambda\right)$, where $\lambda$ is a complex parameter. This is achieved in [8] for Newton's method. 


\section{Equation of critical and additional fixed points for the third-order family of iterative methods}

In this section, we give the equations for critical points and for additional fixed points, if any, for the iterative methods $M_{p, \theta, c}$, when $p$ is a generic polynomial.

Let $p$ be a generic polynomial. The derivative of the iterative method $M_{p, \theta, c}$ is given by

$$
\begin{aligned}
M_{p, \theta, c}^{\prime}(z)= & -\left(\frac{L_{p}^{\prime}(z)}{\left[2\left(1-\theta L_{p}(z)\right)\right]^{2}}+2 c L_{p}(z) L_{p}^{\prime}(z)\right) u_{p}(z) \\
& -\left(1+\frac{L_{p}(z)}{2\left(1-\theta L_{p}(z)\right)}+c\left[L_{p}(z)\right]^{2}\right) u_{p}^{\prime}(z) .
\end{aligned}
$$

Therefore, if $p(z)=0$ and $p^{\prime}(z) \neq 0$, then $M_{p, \theta, c}^{\prime}(z)=0$. Hence, the roots of $p$ are superattracting fixed points of $M_{p, \theta, c}$. The solutions of the equation $M_{p, \theta, c}^{\prime}(z)=0$, other than the roots of $p$, are called free critical points.

(1) For Chebyshev's iterative function $C_{p}$, the derivative is given by

$$
C_{p}^{\prime}(z)=\frac{1}{2}\left(\frac{p(z)}{p^{\prime}(z)}\right)^{2}\left(3\left(\frac{p^{\prime \prime}(z)}{p^{\prime}(z)}\right)^{2}-\frac{p^{\prime \prime \prime}(z)}{p^{\prime}(z)}\right) .
$$

Therefore, if $C_{p}^{\prime}(z)=0$ then either $p(z)=0$ (roots of $p$ ) or $3 p^{\prime \prime}(z)^{2}-p(z) p^{\prime \prime \prime}(z)=0$. The solutions of $3 p^{\prime \prime}(z)^{2}-p^{\prime}(z) p^{\prime \prime \prime}(z)=0$ that are not solutions of $p(z)=0$, if any, are the free critical points of Chebyshev's iterative method. For example, Chebyshev's iterative method has no free critical points when it is applied to the quadratic polynomial $p_{\mu}$ and has $c_{1}, c_{2}= \pm \sqrt{(A-1) / 15}$ as its free critical points when it is applied to the cubic polynomial $p_{A}$ (see [33]).

(2) For Halley's iterative method $H_{p}(z)$, the derivative is given by

$$
\begin{aligned}
H_{p}^{\prime}(z) & =[p(z)]^{2} \frac{3\left[p^{\prime \prime}(z)\right]^{2}-2 p^{\prime}(z) p^{\prime \prime \prime}(z)}{\left(2\left[p^{\prime}(z)\right]^{2}-p(z) p^{\prime \prime}(z)\right)^{2}} \\
& =-2[p(z)]^{2}\left[p^{\prime}(z)\right]^{2} \frac{S(p)(z)}{\left(2\left[p^{\prime}(z)\right]^{2}-p(z) p^{\prime \prime}(z)\right)^{2}},
\end{aligned}
$$

where

$$
S(p)(z)=\frac{p^{\prime \prime \prime}(z)}{p^{\prime}(z)}-\frac{3}{2}\left(\frac{p^{\prime \prime}(z)}{p^{\prime}(z)}\right)^{2}
$$

is the Schwarzian derivative of $p(z)$. Therefore, if $H_{p}^{\prime}(z)=0$, then either $p(z)=0$ (roots of $p$ ) or $3 p^{\prime \prime}(z)^{2}-2 p^{\prime}(z) p^{\prime \prime \prime}(z)=0$. Solutions of $3 p^{\prime \prime}(z)^{2}-2 p^{\prime}(z) p^{\prime \prime \prime}(z)=0$ that are not roots of $p(z)$, if any, are the free critical points of Halley's iteration 
function. For example, Halley's iterative method has no free critical points when it is applied to the quadratic polynomial $p_{\mu}$ and has $c_{1}, c_{2}= \pm \sqrt{(A-1) / 6}$ as free critical points when it is applied to the cubic polynomial $p_{A}$. A study of the parameter space for Halley's iterative method associated to the polynomial $p_{A}$ was done in [31].

(3) For the super-Halley iterative method, the derivative is given by

$$
S H_{p}^{\prime}(z)=\frac{1}{2}\left(\frac{p(z)}{p^{\prime}(z)}\right)^{2} \frac{p(z) p^{\prime \prime}(z)^{3}-p^{\prime}(z)^{3} p^{\prime \prime \prime}(z)}{\left(p^{\prime}(z)^{2}-p(z) p^{\prime \prime}(z)\right)^{2}} .
$$

Therefore, if $S H_{p}^{\prime}(z)=0$, then either $p(z)=0$ (roots of $p$ ) or $p(z) p^{\prime \prime}(z)^{3}-$ $p^{\prime}(z)^{3} p^{\prime \prime \prime}(z)=0$. The solutions of $p(z) p^{\prime \prime}(z)^{3}-p^{\prime}(z)^{3} p^{\prime \prime \prime}(z)=0$ that are not roots of $p(z)$, if any, are the free critical points of the super-Halley iterative method. The super-Halley iterative method applied to the quadratic polynomial $p_{\mu}$ has no free critical points and when it is applied to the cubic polynomial $p_{A}$ the free critical points are the solutions of the equation

$$
54 z^{6}+54(A-1) z^{4}-216 z^{3}-54(A-1)^{2} z^{2}-6(A-1)^{3}=0 .
$$

(4) For the $c$-iterative methods, the derivative is given by

$$
\begin{aligned}
M_{p, c}^{\prime}(z)= & -\frac{p(z)^{2}}{2 p^{\prime}(z)^{6}}\left[3(2 c-1) p^{\prime}(z)^{2} p^{\prime \prime}(z)^{2}-10 c p(z) p^{\prime \prime}(z)^{3}+p^{\prime}(z)^{3} p^{\prime \prime \prime}(z)\right. \\
& \left.+4 c p(z) p^{\prime}(z) p^{\prime \prime}(z) p^{\prime \prime \prime}(z)\right] .
\end{aligned}
$$

Therefore, for a $c$-iterative method the solutions of the equation

$$
\begin{aligned}
& 3(2 c-1) p^{\prime}(z)^{2} p^{\prime \prime}(z)^{2}-10 c p(z) p^{\prime \prime}(z)^{3} \\
& \quad+p^{\prime}(z)^{3} p^{\prime \prime \prime}(z)+4 c p(z) p^{\prime}(z) p^{\prime \prime}(z) p^{\prime \prime \prime}(z)=0
\end{aligned}
$$

that are not roots of $p$, if any, are the free critical points of the $c$-iterative method.

For the $c$-iterative function applied to the quadratic polynomial $p_{\mu}$, the free critical points are $c_{1}, c_{2}= \pm \sqrt{(15-5 c) c \mu} /(3-c)$ and when it is applied to the cubic polynomial $p_{A}$ the free critical points are the solutions of the equation

$$
\begin{aligned}
& 2(405-108 c) z^{6}+2(243+144 c)(A-1) z^{4}+18\left(3\left(A^{2}+1\right)\right. \\
& \quad+20(2-A) A c-2(3 A+10 c)) z^{2}+144 A c(A-1) z-6(A-1)^{3}=0 .
\end{aligned}
$$

The reason why free critical points are important is due to the following classical result.

THEOREM 5.1 (Fatou-Julia). Let $R$ be a rational map. If $z_{0}$ is an attracting periodic point, then the immediate basin of attraction $B^{*}\left(z_{0}\right)$ contains at least one critical point. 
Thus, as a consequence of this theorem, the existence of attracting periodic orbits interferes with our search for roots of the equation $p(z)=0$ because if this is the case, the orbit of each free critical point must be computed and its set of limit points determined. If the set of limit points of the orbit of some free critical point is not a root of $p$, then it must be an attracting periodic orbit of $M_{p, \theta . c}$.

We notice that the existence of an attracting periodic orbit is not all that interferes with the basins of attraction of the roots of $p(z)$. In some cases, there may exist additional fixed points, that is, fixed points other than the roots of $p$. It is clear that the roots of $p$ are fixed points of $M_{p, \theta, c}$. In fact, as we saw earlier, they are superattracting fixed points.

To find the additional fixed points of the iterative methods $M_{p . \theta . c}$, we must solve the equation $M_{p, \theta, c}(z)=z$ subject to the condition $p(z) \neq 0$.

We have $M_{p, \theta . c}(z)=z$ if and only if

$$
\left(1+\frac{L_{p}(z)}{2\left(1-\theta L_{p}(z)\right)}+c L_{p}(z)^{2}\right) u_{p}(z)=0
$$

Since the roots of $p$ are fixed points of $M_{p, \theta, c}$, we may assume that $p(z) \neq 0$. Thus the additional fixed points are the solutions, other than the roots of $p$, if any, of the equation

$$
\begin{aligned}
2 p^{\prime}(z)^{6} & +(1-2 \theta) p(z) p^{\prime}(z)^{4} p^{\prime \prime}(z) \\
& +2 c p(z)^{2} p^{\prime}(z)^{2} p^{\prime \prime}(z)^{2}-2 c \theta p(z)^{3} p^{\prime \prime}(z)^{3}=0 .
\end{aligned}
$$

(a) For Chebyshev's iterative method, the additional fixed points are the solutions, if any, of the equation $2 p^{\prime}(z)^{2}+p(z) p^{\prime \prime}(z)=0$.

For example, Chebyshev's method applied to the quadratic polynomial $p_{\mu}$ has two additional fixed points given by $z_{1}, z_{2}= \pm \sqrt{\mu / 5}$ and when it is applied to the cubic polynomial $p_{A}$ the additional fixed points are the solutions of the equation $12 z^{4}+9(A-1) z^{2}-3 A z+(A-1)^{2}=0$.

(b) For Halley's iterative method, the additional fixed points are given by the solutions of the equation $p^{\prime}(z)=0$, that is, the additional fixed points are the critical points of $p$. Since

$$
H_{p}^{\prime}(z)=\frac{p(z)^{2}\left(3 p^{\prime \prime}(z)^{2}-2 p^{\prime}(z) p^{\prime \prime \prime}(z)\right)}{\left(-2 p^{\prime}(z)^{2}-p(z) p^{\prime \prime}(z)\right)^{2}}
$$

if $p\left(z_{0}\right) \neq 0$ and $p^{\prime}\left(z_{0}\right) \neq 0$, then $H_{f}^{\prime}\left(z_{0}\right)=3$, or in other words any additional fixed points of Halley's method are repelling fixed points (see [19, Proposition 1]).

(c) For the super-Halley iterative method, the additional fixed points are the solutions of the polynomial equation $2 p^{\prime}(z)^{2}-p(z) p^{\prime \prime}(z)=0$. 
The super-Halley method applied to the quadratic polynomial $p_{\mu}$ has two additional fixed points $z_{1}, z_{2}= \pm \sqrt{-\mu / 3}$ and when it is applied to the cubic polynomial $p_{A}$, the additional fixed points are the solutions of the equation

$$
9 z^{6}+9(A-1) z^{4}-36 A z^{3}-9(A-1)^{2} z^{2}-(A-1)^{3}=0 .
$$

(d) For the $c$-methods, the additional fixed points are the solutions of another polynomial equation, namely $2 p^{\prime}(z)^{4}+p(z) p^{\prime}(z)^{2} p^{\prime \prime}(z)+2 c p(z)^{2} p^{\prime \prime}(z)^{2}=0$.

When the $c$-methods are applied to the quadratic polynomial $p_{\mu}$, we have four free fixed points

and

$$
z_{1}, z_{2}= \pm \frac{\sqrt{2} \sqrt{(c+5)(1+2 c+\sqrt{1-16 c}) \mu}}{2 c+10}
$$

$$
z_{3}, z_{4}= \pm \frac{\sqrt{2} \sqrt{(c+5)(1+2 c-\sqrt{1-16 c}) \mu}}{2 c+10}
$$

and when they are applied to the cubic polynomial $p_{A}$, the additional fixed points are the solutions of the equation

$$
\begin{aligned}
36(3+c) z^{8} & +(153+72 c)(A-1) z^{6}-(27+72 c) A z^{5} \\
& +(75+36 c)(A-1)^{2} z^{4}-(18-72 c) A(A-1) z^{3} \\
& +\left(15(A-1)^{3}+36 A^{2} c\right) z^{2}-3 A(A-1)^{2} z-(A-1)^{4}=0 .
\end{aligned}
$$

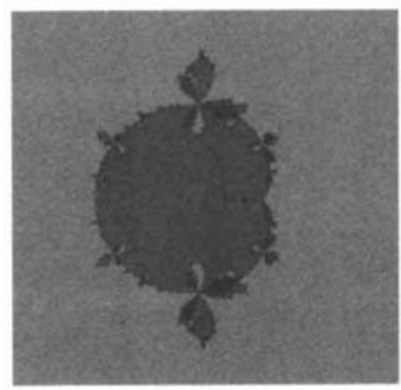

Chebyshev's method

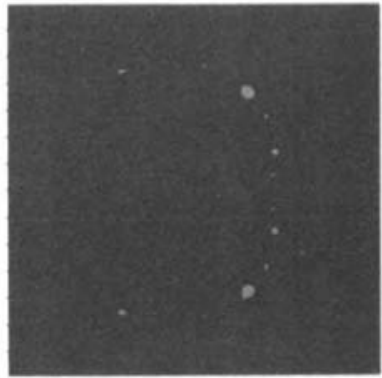

Halley's method

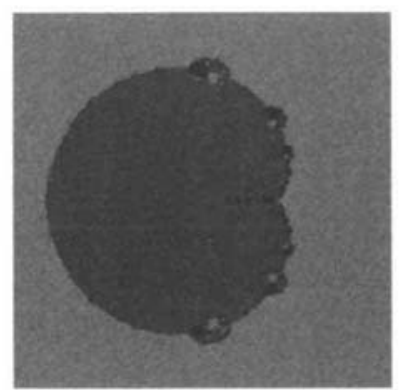

Newton's method

FIGURE 5. The regions in parameter space $A \in[-2,2] \times[-2,2]$ in which the corresponding critical points are attracted to some of the roots of the polynomial $p_{A}$ when the indicated iterative methods are applied to it. 


\section{Conclusions}

To summarize, in this paper we study a large class of third-order methods from the point of view of their dynamics. For each method, we establish its conjugacy classes and find explicit formulations for both its additional fixed points and its free critical points.

TABLE 1. $x_{0}=1.5, x^{*}=2, l_{\infty}-$ error, $n=100$.

\begin{tabular}{|c|c|c|c|c|}
\hline Iterations & Newton & Chebyshev & Halley & $c=2$ \\
\hline 1 & 0.1826 & 0.1474 & 0.0408 & 0.16206 \\
\hline 2 & 0.0146 & 0.0036 & $2.59 \times 10^{-5}$ & $3.86 \times 10^{-4}$ \\
\hline 3 & $1.06 \times 10^{-4}$ & $2.31 \times 10^{-7}$ & $4.86 \times 10^{-13}$ & $1.23 \times 10^{-12}$ \\
\hline 4 & $1.46 \times 10^{-8}$ & $8.41 \times 10^{-13}$ & 0 & 0 \\
\hline 5 & $1.24 \times 10^{-12}$ & 0 & & \\
\hline 6 & 0 & & & \\
\hline
\end{tabular}

We finalize with an application where the studied methods are applied to quadratic equations of the type

$$
F(x)=x^{T} A x+B x+C=0,
$$

where $\operatorname{dim}(A)=(n \times n) \times n, \operatorname{dim}(B)=n \times n$ and $\operatorname{dim}(C)=\operatorname{dim}(x)=n$.

In this case, the second Fréchet derivative is constant, $F^{\prime \prime}(x)=A+A^{T}$.

The above kind of equations may come from the discretization of equilibrium problems, where interacting forces between particles determine the output. The discretization of the Laplacian using divided differences also gives quadratic equations [21]. Other interesting examples may be found in Fredholm equations [18] as

$$
x(s)=f(s)+\lambda \int_{0}^{1} e^{t+s} x(t)^{2} d t .
$$

However, the actual case we are going to analyze will have an exact solution in order to make the evaluation of errors easier. We randomly generate $A$ and $B$, and then we determine $C$ such that $X^{*}(i)=2, i=1, \ldots, n$, is a solution of (6.1).

In Table 1 the dimension is $n=100$.

\section{Acknowledgements}

SA's and SB's research was supported in part by the Spanish grants MTM200407114 and 00675/PI/04. Part of SP's work was supported by Fondecyt Grants $\# 1000098$ and \#1020711 and by Dicyt Grant \#0233 P.S. 


\section{References}

[1] S. Amat and S. Busquier, "Third-order iterative methods under Kantorovich conditions", Appl. Math. Appl., to appear.

[2] S. Amat, S. Busquier and J. M. Gutiérrez, "Geometric constructions of iterative functions to solve nonlinear equations", J. Comput. Appl. Math. 157 (2003) 197-205.

[3] S. Amat, S. Busquier, D. El Kebir and J. Molina, "A fast Chebyshev's method for quadratic equations", Appl. Math. Comput. 148 (2004) 461-471.

[4] S. Amat, S. Busquier and S. Plaza, "Dynamics of a family of third-order iterative methods that do not require using second derivative", Appl. Math. Comput. 154 (2004) 735-746.

[5] N. Argiropoulos, V. Drakopoulos and A. Böhm, "Julia and Mandelbrot-like sets for higher order König rational iterative maps", in Fractal Frontiers (eds. M. M. Novak and T. G. Dewey), (World Scientific, Singapore, 1997) 169-178.

[6] I. K. Argyros, "Quadratic equations and applications to Chandrasekhar's and related equations", Bull. Austral. Math. Soc. 32 (1985) 275-292.

[7] I. K. Argyros and D. Chen, "Results on the Chebyshev method in Banach spaces", Proyecciones 12 (1993) 119-128.

[8] D. C. Arney and B. T. Robinson, "Exhibiting chaos and fractals with a microcomputer", Comput. Math. Appl. 19 (1990) 1-11.

[9] P. Blanchard, "Complex analytic dynamics on the Riemann sphere", Bull. Amer. Math. Soc. 11 (1984) 85-141.

[10] X. Buff and C. Henriksen, "On the König root-finding algorithms", Nonlinearity 16 (2003) 9891015.

[11] V. F. Candela and A. Marquina, "Recurrence relations for rational cubic methods I. The Halley method", Computing 44 (1990) 169-184.

[12] V.F. Candela and A. Marquina, "Recurrence relations for rational cubic methods II. The Chebyshev method", Computing 45 (1990) 355-367.

[13] A. Cayley, "The Newton-Fourier imaginary problem", Amer. J. Math. 2 (1879) 97.

[14] A. Cayley, "On the Newton-Fourier imaginary problem", Proc. Cambridge Phil. Soc. 3 (1880) 231-232.

[15] D. Chen, "Ostrowski-Kantarovich theorem and S-order of convergence of Halley method in Banach spaces”, Comment. Math Univ. Carolina 58 (1993) 215-224.

[16] D. Chen, I. K. Argyros and Q. S. Qian, "A local convergence theorem for the super-Halley method in Banach spaces", Appl. Math. Lett. 7 (1994) 49-52.

[17] J. H. Curry, L. Garnett and D. Sullivan, "On the iteration of a rational function: computer experiment with Newton's method", Comm. Math. Phys. 91 (1983) 267-277.

[18] H. T. Davis, Introduction to nonlinear differential and integral equations (Dover, New York, 1962).

[19] V. Drakopoulos, "How is the dynamics of König iterative method affected by their additional fixed points?", Fractals 7 (1999) 327-334.

[20] V. Drakopoulos, "Are there any Julia sets for Laguerre iterative method?", Comput. Math. Appl. 46 (2003) 1201-1210.

[21] J. A. Ezquerro, J. M. Gutiérrez, M. A. Hernández and M. A. Salanova, "The super-Halley method and partial differential equations", in $X V$ Congress on Differential Equations and Applications/ $V$ Congress on Applied Mathematics (Vigo, 1997), (Spanish), (Univ. Vigo, Vigo, 1998) 713-718.

[22] J. A. Ezquerro, J. M. Gutiérrez, M. A. Hernández and M. A. Salanova, "Chebyshev-like methods and quadratic equations", Rev. Anal. Numer. Theor. Approx. 28 (1999) 23-35.

[23] J. M. Gutiérrez and M. A. Hernández, "A family of Chebyshev-Halley type methods in Banach spaces", Bull. Austral. Math. Soc. 55 (1997) 113-130.

[24] J.M. Gutiérrez and M. A. Hernández, "An acceleration of Newton's method: super-Halley method", 
Appl. Math. Comput. 117 (2001) 223-239.

[25] J. M. Gutiérrez, M. A. Hernández and M. A. Salanova, "Quadratic equations in Banach spaces", Numer. Func. Anal. Optim. 7 (1996) 113-121.

[26] M. A. Hernández, "A family of Chebyshev-Halley type methods", Internat. J. Comput. Math. 47 (1993) 59-63.

[27] K. Kneisl, "Julia sets for the super-Newton method, Cauchy's method and Halley's method", Chaos 11 (2001).

[28] J. Milnor, Dynamics in one complex variable: introductory lectures (Vieweg, Braunschweig, 1999).

[29] S. Morosawa, Y. Nishimura, M. Taniguchi and T. Ueda, Holomorphic dynamics (Cambridge University Press, Cambridge, 1999).

[30] H. O. Peitgen (ed.), Newton's method and dynamical systems (Kluwer Academic Publishers, Dordrecht, 1989).

[31] G. E. Roberts and J. Horgan-Kobelski, "Newton's versus Halley's method: an approach via complex dynamics", Internat. J. Bifur. Chaos Appl. Sci. Engrg. 14 (2004) 3459-3475.

[32] E. O. Schröder, "On infinitely many algorithms for solving equations", Math. Ann. 2 (1870) 317-265; Translated by G. W. Stewart, 1992 (this report is available by anonymous ftp from thales.cs.umd.edu in the directory pub/reports).

[33] E. Vrscay, "Julia sets and Mandelbrot-like sets associated with higher order Schröder rational iterative methods: a computer assisted study", Math. Comput. 46 (1986) 151-169.

[34] E. Vrscay and W. Gilbert, "Extraneous fixed points, basin boundary and chaotic dynamics for Schröder and König rational iterative methods", Numer. Math. 52 (1988) 1-16. 